\title{
ERRATUM: “THE EIGHTH DATA RELEASE OF THE SLOAN DIGITAL SKY SURVEY: FIRST DATA FROM SDSS-III” (2011, ApJS, 193, 29)
}

Hiroaki Aihara ${ }^{1}$, Carlos Allende Prieto $^{2,3}$, Deokkeun An ${ }^{4}$, Scott F. Anderson ${ }^{5}$, Éric Aubourg ${ }^{6,7}$, Eduardo Balbinot ${ }^{8,9}$, Timothy C. Beers ${ }^{10}$, Andreas A. Berlind ${ }^{11}$, Steven J. Bickerton ${ }^{12}$, Dmitry Bizyaev ${ }^{13}$, Michael R. Blanton ${ }^{14}$, John J. Bochanski ${ }^{15}$, Adam S. Bolton ${ }^{16}$, Jo Bovy ${ }^{14}$, W. N. Brandt ${ }^{15}, 17$, J. Brinkmann ${ }^{13}$, Peter J. Brown ${ }^{16}$, Joel R. Brownstein ${ }^{16}$, Nicolas G. Busca ${ }^{6}$, Heather Campbell ${ }^{18}$, Michael A. Carr ${ }^{12}$, Yanmei Chen ${ }^{19}$, Cristina Chiappini ${ }^{9}, 20,21$, Johan Comparat ${ }^{22}$, Natalia Connolly ${ }^{23}$, Marina Cortes ${ }^{24}$, Rupert A. C. Croft ${ }^{25}$, Antonio J. Cuesta ${ }^{26}$, Luiz N. da Costa ${ }^{9,27}$, James R. A. DavenPort ${ }^{5}$, Kyle Dawson $^{16}$, Saurav Dhital ${ }^{11}$, Anne Ealet ${ }^{28}$, Garrett L. Ebelke ${ }^{13,29}$, Edward M. Edmondson ${ }^{18}$, Daniel J. Eisenstein ${ }^{30,31}{ }^{3}$ STePhanie Escoffier $^{28}$, Massimiliano Esposito ${ }^{2,3}$, Michael L. Evans ${ }^{5}$, Xiaohui Fan ${ }^{30}$, Bruno Femenía Castella ${ }^{2,3}$, Andreu Font-Ribera ${ }^{32}$,

Peter M. Frinchaboy ${ }^{33}$, Jian Ge ${ }^{34}$, Bruce A. Gillespie ${ }^{13}$, G. Gilmore ${ }^{35}$, Jonay I. GonZález Hernández ${ }^{2}$, J. Richard GotT ${ }^{12}$, Andrew Gould ${ }^{36}$, Eva K. Grebel ${ }^{37}$, James E. GunN ${ }^{12}$, Jean-Christophe Hamilton ${ }^{6}$, Paul Harding ${ }^{38}$, David W. Harris ${ }^{16}$, Suzanne L. Hawley ${ }^{5}$, Frederick R. Hearty ${ }^{39}$, Shirley Ho ${ }^{24}$, David W. Hogg ${ }^{14}$, Jon A. Holtzman ${ }^{29}$,

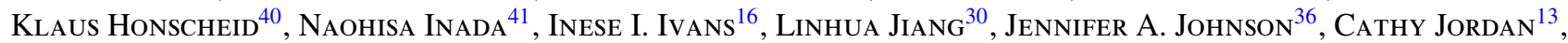
Wendell P. Jordan ${ }^{13,29}$, Eyal A. Kazin ${ }^{14}$, David KirkbY ${ }^{42}$, Mark A. Klaene ${ }^{13}$, G. R. Knapp ${ }^{12}$, Jean-Paul Kneib ${ }^{22}$, C. S. KochaneK ${ }^{36}$, Lars Koesterke ${ }^{43}$, Juna A. Kollmeier ${ }^{44}$, Richard G. Kron ${ }^{45,46}$, Hubert Lampeitl ${ }^{18}$, Dustin Lang $^{12}$, Jean-Marc Le GofF ${ }^{7}$, Young Sun LeE ${ }^{10}$, Yen-Ting Lin ${ }^{1,47}$, Daniel C. Long ${ }^{13}$, Craig P. Loomis ${ }^{12}$, Sara Lucatello ${ }^{48}$,

Britt Lundgren ${ }^{26}$, Robert H. Lupton ${ }^{12}$, Zhibo Ma ${ }^{38}$, Nicholas MacDonald ${ }^{5}$, Suvrath Mahadevan ${ }^{15,49}$,

Marcio A. G. Maia ${ }^{9,27}$, Martin Makler ${ }^{9,50}$, Elena Malanushenko ${ }^{13}$, Viktor Malanushenko $^{13}$, Rachel Mandelbaum $^{12}$, Claudia Maraston ${ }^{18}$, Daniel Margala ${ }^{42}$, Karen L. Masters ${ }^{18}$, Cameron K. McBride $^{11}$, Peregrine M. McGehee ${ }^{51}$, Ian D. McGreer ${ }^{30}$, Brice Ménard ${ }^{1,52,53}$, Jordi Miralda-Escudé ${ }^{54,55}$, Heather L. Morrison $^{38}$, F. Mullally ${ }^{12,56}$, Demitri Muna ${ }^{14}$, Jeffrey A. Munn ${ }^{57}$, Hitoshi Murayama ${ }^{1}$, Adam D. Myers ${ }^{58}$, Tracy Naugle ${ }^{13}$, Angelo Fausti Neto ${ }^{8,9}$, Duy Cuong NGuYen ${ }^{34}$, Robert C. Nichol ${ }^{18}$, Robert W. O'CONNell ${ }^{39}$, Ricardo L. C. Ogando ${ }^{9,27}$,

Matthew D. Olmstead ${ }^{16}$, Daniel J. Oravetz ${ }^{13}$, Nikhil Padmanabhan ${ }^{26}$, Nathalie Palanque-Delabrouille ${ }^{7}$, Kaike Pan ${ }^{13}$, Parul Pandey ${ }^{16}$, Isabelle Pâris ${ }^{59}$, Will J. Percival ${ }^{18}$, Patrick Petitjean ${ }^{59}$, Robert Pfaffenberger ${ }^{29}$, Janine Pforr $^{18}$, Stefanie Phleps ${ }^{60}$, Christophe Pichon ${ }^{59}$, Matthew M. Pieri ${ }^{36,61}$, Francisco Prada ${ }^{62}$,

Adrian M. Price-Whelan ${ }^{14}$, M. Jordan RaddicK ${ }^{53}$, Beatriz H. F. Ramos ${ }^{9,27}$, CÉline Reylé63 ${ }^{63}$ James Rich $^{7}$, Gordon T. Richards ${ }^{64}$, Hans-Walter Rix ${ }^{65}$, Annie C. Robin ${ }^{63}$, Helio J. Rocha-Pinto ${ }^{9,66}$, Constance M. Rockosi ${ }^{67}$,

Natalie A. Roe ${ }^{24}$, Emmanuel Rollinde ${ }^{59}$, Ashley J. Ross ${ }^{18}$, Nicholas P. Ross ${ }^{24}$, Bruno M. Rossetto ${ }^{9,66}$,

Ariel G. Sánchez ${ }^{60}$, Conor Sayres ${ }^{5}$, David J. Schlegel ${ }^{24}$, Katharine J. Schlesinger ${ }^{36}$, Sarah J. Schmidt ${ }^{5}$,

Donald P. Schneider ${ }^{15,49}$, Erin Sheldon ${ }^{68}$, Yiping Shu ${ }^{16}$, Jennifer Simmerer ${ }^{16}$, Audrey E. Simmons ${ }^{13}$,

Thirupathi Sivarani ${ }^{34,69}$, STephanie A. Snedden ${ }^{13}$, Jennifer S. Sobeck ${ }^{46}$, Matthias Steinmetz $^{20}$, Michael A. Strauss ${ }^{12}$, Alexander S. Szalay ${ }^{53}$, Masayuki Tanaka ${ }^{1}$, Aniruddha R. Thakar ${ }^{53}$, Daniel Thomas ${ }^{18}$, Jeremy L. Tinker ${ }^{14}$, Benjamin M. Tofflemire ${ }^{5}$, Rita Tojeiro ${ }^{18}$, Christy A. Tremonti ${ }^{19}{ }^{2}$ Jan Vandenberg $^{53}$, M. Vargas Magaña ${ }^{6}$, Licia Verde ${ }^{54,55}$, Nicole P. Vogt ${ }^{29}$, David A. Wake ${ }^{26}$, Ji Wang ${ }^{34}$, Benjamin A. Weaver ${ }^{14}$, David H. Weinberg ${ }^{36}$, Martin White $^{70}$, Simon D. M. White ${ }^{71}$, Brian YannY ${ }^{45}$, Naoki Yasuda ${ }^{1}$, Christophe YeChe $^{7}$, and Idit Zehavi ${ }^{38}$

${ }^{1}$ Institute for the Physics and Mathematics of the Universe, The University of Tokyo, Kashiwa 277-8583, Japan

${ }^{2}$ Instituto de Astrofísica de Canarias, E38205 La Laguna, Tenerife, Spain

${ }^{3}$ Departamento de Astrofísica, Universidad de La Laguna, 38206, La Laguna, Tenerife, Spain

${ }^{4}$ Department of Science Education, Ewha Womans University, Seoul 120-750, Republic of Korea

${ }^{5}$ Department of Astronomy, University of Washington, Seattle, WA 98195, USA

${ }^{6}$ Astroparticule et Cosmologie (APC), Université Paris-Diderot, 75205 Paris Cedex 13, France

${ }^{7}$ CEA, Centre de Saclay, Irfu/SPP, F-91191 Gif-sur-Yvette, France

${ }^{8}$ Instituto de Física, UFRGS, Porto Alegre, RS 91501-970, Brazil

${ }^{9}$ Laboratório Interinstitucional de e-Astronomia (LIneA), Rio de Janeiro, RJ 20921-400, Brazil

${ }^{10}$ Department of Physics \& Astronomy and JINA: Joint Institute for Nuclear Astrophysics, Michigan State University, E. Lansing, MI 48824, USA

${ }^{11}$ Department of Physics and Astronomy, Vanderbilt University, Nashville, TN 37235, USA

${ }^{12}$ Department of Astrophysical Sciences, Princeton University, Princeton, NJ 08544, USA ${ }_{13}$ Apache Point Observatory, Sunspot, NM 88349, USA

${ }^{14}$ Center for Cosmology and Particle Physics, New York University, New York, NY 10003, USA

${ }^{15}$ Department of Astronomy and Astrophysics, 525 Davey Laboratory, The Pennsylvania State University, University Park, PA 16802, USA

${ }^{16}$ Department of Physics and Astronomy, University of Utah, Salt Lake City, UT 84112, USA

${ }^{17}$ Institute for Gravitation and the Cosmos, The Pennsylvania State University, University Park, PA 16802, USA

${ }^{18}$ Institute of Cosmology and Gravitation (ICG), Dennis Sciama Building, University of Portsmouth, Portsmouth, PO1 3FX, UK

${ }^{19}$ Department of Astronomy, University of Wisconsin-Madison, Madison, WI 53703, USA ${ }^{20}$ Astrophysical Institute Potsdam, 14482 Potsdam, Germany

${ }^{21}$ Istituto Nazionale di Astrofisica, 34143 Trieste, Italy

${ }^{22}$ Laboratoire d'Astrophysique de Marseille, CNRS-Université de Provence, 13388 Marseille Cedex 13, France

${ }^{23}$ Department of Physics, Hamilton College, Clinton, NY 13323, USA

${ }^{24}$ Lawrence Berkeley National Laboratory, Berkeley, CA 94720, USA

${ }^{25}$ Bruce and Astrid McWilliams Center for Cosmology, Carnegie Mellon University, Pittsburgh, PA 15213, USA 


\author{
${ }^{26}$ Yale Center for Astronomy and Astrophysics, Yale University, New Haven, CT 06520, USA \\ ${ }^{27}$ Observatório Nacional, Rio de Janeiro, RJ 20921-400, Brazil \\ ${ }^{28}$ Centre de Physique des Particules de Marseille, Aix-Marseille Université, CNRS/IN2P3, Marseille, France \\ ${ }^{29}$ Department of Astronomy, MSC 4500, New Mexico State University, Las Cruces, NM 88003, USA \\ ${ }^{30}$ Steward Observatory, Tucson, AZ 85721, USA \\ ${ }^{31}$ Harvard College Observatory, Cambridge, MA 02138, USA \\ ${ }^{32}$ Institut de Ciències de l'Espai (IEEC/CSIC), Campus UAB, E-08193 Bellaterra, Barcelona, Spain \\ ${ }^{33}$ Department of Physics \& Astronomy, Texas Christian University, Fort Worth, TX 76129, USA \\ ${ }^{34}$ Department of Astronomy, University of Florida, Bryant Space Science Center, Gainesville, FL 32611-2055, USA \\ ${ }^{35}$ Institute of Astronomy, University of Cambridge, Cambridge, CB3 OHA, UK \\ 36 Department of Astronomy, Ohio State University, Columbus, OH 43210, USA \\ ${ }^{37}$ Astronomisches Rechen-Institut, Zentrum für Astronomie der Universität Heidelberg, 69120 Heidelberg, Germany \\ ${ }^{38}$ Department of Astronomy, Case Western Reserve University, Cleveland, OH 44106, USA \\ ${ }^{39}$ Department of Astronomy, University of Virginia, Charlottesville, VA 22904-4325, USA \\ ${ }^{40}$ Department of Physics, Ohio State University, Columbus, OH 43210, USA \\ ${ }^{41}$ Research Center for the Early Universe, Graduate School of Science, The University of Tokyo, Bunkyo, Tokyo 113-0033, Japan \\ ${ }^{42}$ Department of Physics and Astronomy, University of California, Irvine, CA 92697, USA \\ ${ }^{43}$ Texas Advanced Computer Center, University of Texas, Austin, TX 78758-4497, USA \\ ${ }^{44}$ Observatories of the Carnegie Institution of Washington, Pasadena, CA 91101, USA \\ ${ }^{45}$ Fermi National Accelerator Laboratory, Batavia, IL 60510, USA \\ ${ }^{46}$ Department of Astronomy and Astrophysics, University of Chicago, Chicago, IL 60637, USA \\ ${ }^{47}$ Institute of Astronomy and Astrophysics, Academia Sinica, Taipei 10617, Taiwan \\ ${ }^{48}$ INAF, Osservatorio Astronomico di Padova, 35122 Padova, Italy \\ ${ }^{49}$ Center for Exoplanets and Habitable Worlds, 525 Davey Laboratory, Pennsylvania State University, University Park, PA 16802, USA \\ ${ }^{50}$ ICRA-Centro Brasileiro de Pesquisas Físicas, Urca, Rio de Janeiro, RJ 22290-180, Brazil \\ ${ }^{51}$ IPAC, MS 220-6, California Institute of Technology, Pasadena, CA 91125, USA \\ ${ }^{52}$ CITA, University of Toronto, University of Toronto, Toronto, ON M5S 3H8, Canada \\ ${ }^{53}$ Center for Astrophysical Sciences, Department of Physics and Astronomy, Johns Hopkins University, \\ Baltimore, MD 21218, USA \\ ${ }^{54}$ Institució Catalana de Recerca i Estudis Avançats, Barcelona, Spain \\ 55 Institut de Ciències del Cosmos, Universitat de Barcelona/IEEC, Barcelona 08028, Spain \\ ${ }^{56}$ SETI Institute/NASA Ames Research Center, Moffett Field, CA 94035, USA \\ ${ }^{57}$ US Naval Observatory, Flagstaff Station, Flagstaff, AZ 86001-8521, USA \\ ${ }^{58}$ Department of Astronomy, University of Illinois, Urbana, IL 61801, USA \\ ${ }^{59}$ Université Paris 6, Institut d'Astrophysique de Paris, UMR7095-CNRS, F-75014 Paris, France \\ ${ }^{60}$ Max-Planck-Institut für Extraterrestrische Physik, 85748 Garching, Germany \\ ${ }^{61}$ Center for Astrophysics and Space Astronomy, University of Colorado, 389 UCB, Boulder, CO 80309, USA \\ 62 Instituto de Astrofisica de Andalucia (CSIC), E-18008, Granada, Spain \\ ${ }^{63}$ Institut Utinam, Observatoire de Besançon, Université de Franche-Comté, BP1615, F-25010 Besançon Cedex, France \\ ${ }^{64}$ Department of Physics, Drexel University, Philadelphia, PA 19104, USA \\ ${ }^{65}$ Max-Planck-Institut für Astronomie, D-69117 Heidelberg, Germany \\ ${ }^{66}$ Observatório do Valongo, Universidade Federal do Rio de Janeiro, 20080-090 Rio de Janeiro, Brazil \\ ${ }^{67}$ UCO/Lick Observatory, University of California, Santa Cruz, Santa Cruz, CA 95064, USA \\ ${ }^{68}$ Bldg. 510 Brookhaven National Laboratory Upton, NY 11973, USA \\ ${ }^{69}$ Indian Institute of Astrophysics, II Block, Koramangala, Bangalore 560 034, India \\ ${ }^{70}$ Physics Department, University of California, Berkeley, CA 94720, USA \\ ${ }^{71}$ Max-Planck-Institut für Astrophysik, D-85748 Garching, Germany \\ Received 2011 June 28; published 2011 August 5
}

Section 3.5 of Aihara et al. (2011) described various sources of systematic error in the astrometry of the imaging data of the Sloan Digital Sky Survey (SDSS). In addition to these sources of error, there is an additional and more serious error, which introduces a large systematic shift in the astrometry over a large area around the north celestial pole. The region has irregular boundaries but in places extends as far south as declination $\delta \approx 41^{\circ}$. The sense of the shift is that the positions of all sources in the affected area are offset by roughly 250 mas in a northwest direction. We have updated the SDSS online documentation ${ }^{72}$ to reflect these errors, and to provide detailed quality information for each SDSS field.

In the Seventh Data Release of the SDSS (Abazajian et al. 2009), the astrometric calibration was performed with respect to the second data release of the United States Naval Observatory (USNO) CCD Astrograph Catalog (UCAC2; Zacharias et al. 2004), and a supplemental set of UCAC results in an internal USNO product known as "r14." The UCAC r14 data were used for declinations northward of approximately $40^{\circ}-50^{\circ}$ depending on right ascension. However, in the SDSS Eighth Data Release (DR8), we did not use the UCAC r14 catalog at high declination, but instead used the USNO-B catalog (Monet et al. 2003). The UCAC and USNO-B systems have a relative systematic offset of about 250 mas. The UCAC system is in much better agreement with the Tycho- 2 system (Høg et al. 2000) of the Hipparcos astrometric satellite.

We have performed a detailed comparison of the large-scale differences in astrometry between the SDSS DR8 and the UCAC catalogs. In the regions not covered by UCAC2 (starting northward of roughly $41^{\circ}$ declination), the DR8 astrometry is offset in the mean 240 mas to the north and 50 mas to the west relative to the r 14 catalog. On scales of about 0.25 , the rms scatter around this offset is about 80 mas in the declination direction and 94 mas in the right ascension direction. Some of that scatter is coherent on larger

\footnotetext{
$72 \mathrm{http} / / / \mathrm{www} . \mathrm{sdss} 3$. org
} 

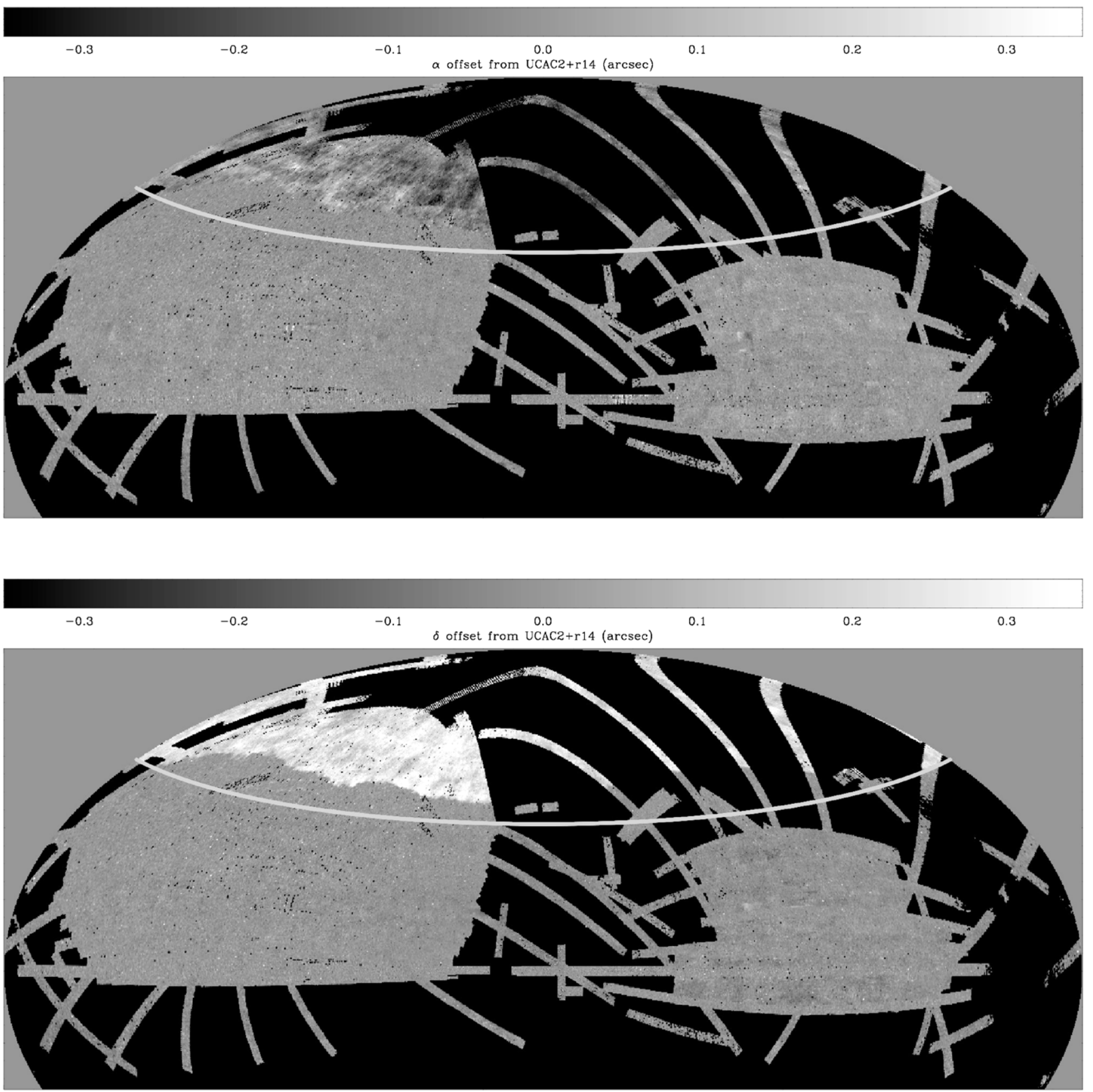

Figure 1. Difference between the coordinates of stars in the SDSS DR8 and those in UCAC2 (mostly south of $\delta=41^{\circ}$ ) and $\mathrm{r} 14\left(\right.$ mostly north of $\delta=41^{\circ}$ ), represented in gray scale as a function of right ascension and declination. The top panel shows differences in right ascension and the bottom panel shows differences in declination. The differences have been smoothed on scales of about 0.25 . The right ascension residuals are multiplied by $\cos \delta$ so that they are in units of proper angular distance. The residuals are shown in an Aitoff projection in equatorial coordinates. The gray line shows $\delta=41^{\circ}$. Black areas are outside the DR8 coverage.

scales; if we unsharp-mask by subtracting off the residual field smoothed with a Gaussian (FWHM $=3^{\circ}$ ), the remaining rms scatter is about 60 mas in either direction. A similar analysis south of $\delta=41^{\circ}$ yields very small offsets (less than 10 mas) between DR8 and UCAC2, with closer to the expected level of scatter (40 mas), and with no large-scale coherence to the scatter. These quantities include the effects of the systematic errors described in Section 3.5 of Aihara et al. (2011).

Figure 1 shows the nature and pattern of the DR8 offsets relative to the UCAC and r14 catalogs as a function of position on the sky.

The effect on the proper motions published in DR8 of the new errors described here is relatively small, because the proper motions in both DR7 and DR8 are calculated relative to USNO-B anyway (using local recalibrations). However, as noted in Section 3.5, the other errors in astrometry do have an effect on the proper motions. In the region with large astrometric errors in DR8, there is no

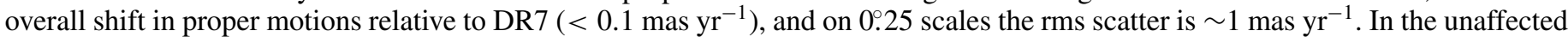

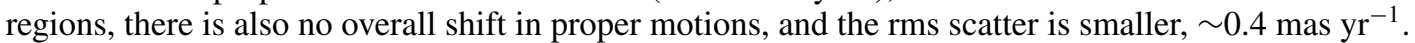


We recommend users requiring correct global astrometry in the affected areas to use DR7 astrometry where available; we provide matches to DR7 in the DR8 Catalog Archive Server (in the photoPrimaryDR7 and photo0bjDR7 tables). We are repairing the errors in the DR8 astrometry and will publish improved astrometric quantities and proper motions.

\section{REFERENCES}

Abazajian, K., Adelman-McCarthy, J. K., Agüeros, M. A., et al. 2009, ApJS, 182, 543

Aihara, H., Allende Prieto, C., An, D., et al. 2011, ApJS, 193, 29

Høg, E., Fabricius, C., Makarov, V. V., et al. 2000, A\&A, 355, L27

Monet, D. G., Levine, S. E., Canzian, B., et al. 2003, AJ, 125, 984

Zacharias, N., Urban, S. E., Zacharias, M. I., et al. 2004, AJ, 127, 3043 